\title{
The ALMA-PILS survey: First detections of deuterated formamide and deuterated isocyanic acid in the interstellar medium
}

\author{
A. Coutens ${ }^{1}$, J. K. Jørgensen ${ }^{2}$, M. H. D. van der Wiel ${ }^{2}$, H. S. P. Müller ${ }^{3}$, J. M. Lykke², P. Bjerkeli ${ }^{2,4}$, T. L. Bourke ${ }^{5}$, \\ H. Calcutt ${ }^{2}$, M. N. Drozdovskaya ${ }^{6}$, C. Favre ${ }^{7}$, E. C. Fayolle ${ }^{8}$, R. T. Garrod ${ }^{9}$, S. K. Jacobsen ${ }^{2}$, N. F. W. Ligterink ${ }^{6}$, \\ K. I. Öberg ${ }^{8}$, M. V. Persson ${ }^{6}$, E. F. van Dishoeck ${ }^{6,10}$, and S. F. Wampfler ${ }^{2}$
}

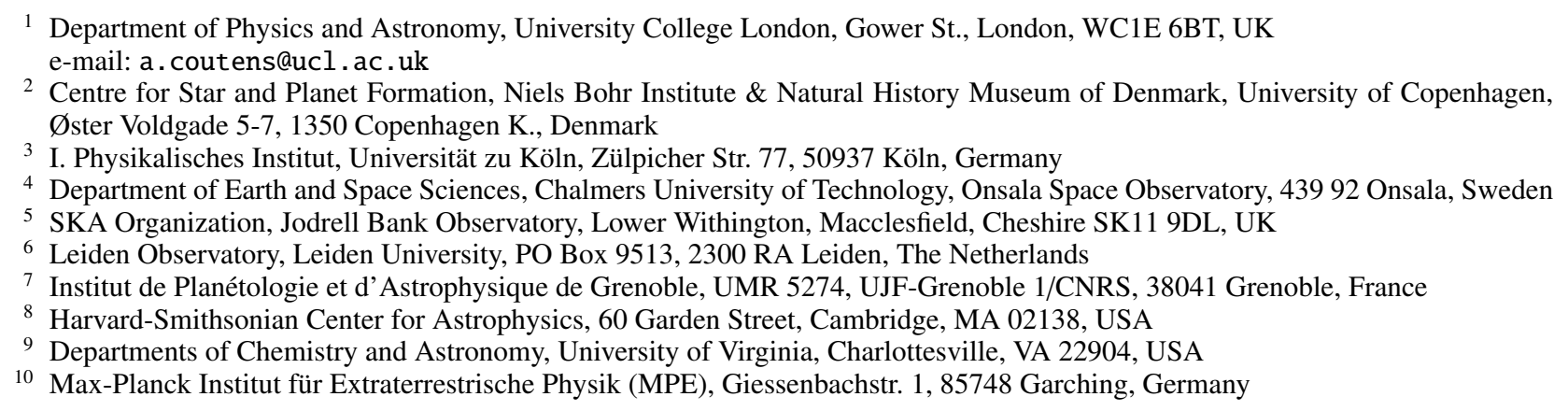

Received 31 March 2016 / Accepted 6 May 2016

\begin{abstract}
Formamide $\left(\mathrm{NH}_{2} \mathrm{CHO}\right)$ has previously been detected in several star-forming regions and is thought to be a precursor for different prebiotic molecules. Its formation mechanism is still debated, however. Observations of formamide, related species, and their isopotologues may provide useful clues to the chemical pathways leading to their formation. The Protostellar Interferometric Line Survey (PILS) represents an unbiased, high angular resolution and sensitivity spectral survey of the low-mass protostellar binary IRAS 16293-2422 with the Atacama Large Millimeter/submillimeter Array (ALMA). For the first time, we detect the three singly deuterated forms of $\mathrm{NH}_{2} \mathrm{CHO}\left(\mathrm{NH}_{2} \mathrm{CDO}\right.$, cis- and trans-NHDCHO), as well as DNCO towards the component $\mathrm{B}$ of this binary source. The images reveal that the different isotopologues are all present in the same region. Based on observations of the ${ }^{13} \mathrm{C}$ isotopologues of formamide and a standard ${ }^{12} \mathrm{C} /{ }^{13} \mathrm{C}$ ratio, the deuterium fractionation is found to be similar for the three different forms with a value of about $2 \%$. The $\mathrm{DNCO} / \mathrm{HNCO}$ ratio is also comparable to the $\mathrm{D} / \mathrm{H}$ ratio of formamide $(\sim 1 \%)$. These results are in agreement with the hypothesis that $\mathrm{NH}_{2} \mathrm{CHO}$ and $\mathrm{HNCO}$ are chemically related through grain-surface formation.
\end{abstract}

Key words. astrochemistry - stars: formation - stars: protostars - ISM: molecules - ISM: individual objects: IRAS 16293-2422 astrobiology

\section{Introduction}

Formamide $\left(\mathrm{NH}_{2} \mathrm{CHO}\right)$, also known as methanamide, contains the amide bond $(-\mathrm{N}-\mathrm{C}(=\mathrm{O})-)$, which plays an important role in the synthesis of proteins. This molecule is a precursor for potential compounds of genetic and metabolic interest (Saladino et al. 2012). Interestingly, it is present in various astrophysical environments: high-mass star-forming regions (e.g. Bisschop et al. 2007; Adande et al. 2013), low-mass protostars (Kahane et al. 2013; López-Sepulcre et al. 2015), shocked regions (Yamaguchi et al. 2012; Mendoza et al. 2014), a translucent cloud (Corby et al. 2015), comets (Bockelée-Morvan et al. 2000; Biver et al. 2014; Goesmann et al. 2015), and even an extragalactic source (Muller et al. 2013).

The formation of formamide is still not clearly understood: several routes have been proposed, both in the gas phase and on the grain surfaces. In the gas phase, many ion-molecule reactions have been ruled out as not sufficiently efficient owing to endothermicity or high-energy barriers (see e.g. Redondo et al. 2014a,b). A neutral-neutral reaction between $\mathrm{H}_{2} \mathrm{CO}$ and $\mathrm{NH}_{2}$ was, however, shown to be barrierless and could account for the abundance of formamide in some sources (Barone et al. 2015). On the grain surface, it has been suggested that formamide forms through the reaction between $\mathrm{HCO}$ and $\mathrm{NH}_{2}$ (Jones et al. 2011; Garrod 2013) and/or hydrogenation of isocyanic acid, HNCO. In particular, the latter suggestion is supported by a strong correlation between the $\mathrm{HNCO}$ and $\mathrm{NH}_{2} \mathrm{CHO}$ abundances in different sources (Bisschop et al. 2007; Mendoza et al. 2014; López-Sepulcre et al. 2015). However, an experiment based on the H-bombardment of $\mathrm{HNCO}$ at low temperature has recently shown that this reaction is not efficient in cold environments (Noble et al. 2015). Instead, other pathways to $\mathrm{HNCO}$ and $\mathrm{NH}_{2} \mathrm{CHO}$ on grains have been suggested, either with or without UV or ion bombardment (see e.g. Kaňuchová et al. 2016, and references therein).

Measurements of isotopic fractionation may help to constrain formation pathways of molecules since isotopic fractionation (especially deuteration) is sensitive to physical conditions such as density and temperature. Until recently, the study of deuteration in solar-type protostars was mainly limited 
to relatively small and abundant molecules, such as $\mathrm{H}_{2} \mathrm{O}$, $\mathrm{HCO}^{+}, \mathrm{HCN}, \mathrm{H}_{2} \mathrm{CO}$, and $\mathrm{CH}_{3} \mathrm{OH}$. Even though the deuterium fractionation is known to be enhanced in low-mass protostars (see e.g. Ceccarelli et al. 2007), measurements of lines of deuterated complex organic molecules (COMs) still require high-sensitivity observations. So far, only deuterated methyl formate and dimethyl ether have been detected towards the lowmass protostar IRAS 16293-2422 (hereafter IRAS 16293) by Demyk et al. (2010) and Richard et al. (2013). With the Atacama Large Millimeter/submillimeter Array (ALMA), it is now possible to search for the isotopologues of complex and less abundant species. In this Letter, we report the first detection of the three singly deuterated forms of formamide, as well as DNCO towards IRAS 16293. These observations mark the first detections of those isotopologues in the interstellar medium.

\section{Observations}

An ALMA unbiased spectral survey of the binary protostar IRAS16293 was recently carried out in the framework of the Protostellar Interferometric Line Survey ${ }^{1}$ (PILS; Jørgensen et al. 2016). The observations were centered on a position at equal distance between the sources A and B that are separated by $\sim 5^{\prime \prime}$. A full description of the survey and the data reduction can be found in Jørgensen et al. (2016). For this work, we use the part of the large spectral survey obtained in Band 7 between $329.15 \mathrm{GHz}$ and $362.90 \mathrm{GHz}$, both with the $12 \mathrm{~m}$ array and the Atacama Compact Array (ACA). The spectral resolution of these observations is $0.244 \mathrm{MHz}$ (i.e. $\sim 0.2 \mathrm{~km} \mathrm{~s}^{-1}$ ). After combining the $12 \mathrm{~m}$ and ACA data, the final spectral line datacubes show a sensitivity that is better than $5 \mathrm{mJy}$ beam ${ }^{-1} \mathrm{~km} \mathrm{~s}^{-1}$. The beam sizes range between $0.4^{\prime \prime}$ and $0.7^{\prime \prime}$. Additional observations in Bands 3 and 6 cover narrow spectral ranges and, consequently, a very limited number of transitions of formamide isotopologues. After the analysis of Band 7, we checked that the results are consistent with these lower frequency observations.

\section{Analysis and results}

To search for the isotopologues of formamide, we use the spectrum extracted at the same position as in Lykke et al. (in prep.), i.e. a position offset by $\sim 0.5^{\prime \prime}$ from the continuum peak of source $\mathrm{B}$ in the south west direction $\left(\alpha_{\mathrm{J} 2000}=16^{\mathrm{h}} 32^{\mathrm{m}} 22.58, \delta_{\mathrm{J} 2000}=\right.$ $\left.-24^{\circ} 28^{\prime} 32.8^{\prime \prime}\right)$. Although the lines are brighter at the position of the continuum peak, the presence of both absorption and emission makes analysis difficult. At the selected position, most of the lines present Gaussian profiles and are relatively bright compared to other positions. In source A, the lines are quite broad, leading to significant line confusion that prevents the search for isotopologues of complex species (e.g. Jørgensen et al. 2012). This Letter is therefore focused on source B only.

Using the CASSIS ${ }^{2}$ software, we identify several unblended lines that can be assigned to the three singly deuterated forms of $\mathrm{NH}_{2} \mathrm{CHO}$ and to $\mathrm{NH}_{2}{ }^{13} \mathrm{CHO}, \mathrm{DNCO}$, and $\mathrm{HN}^{13} \mathrm{CO}$ (see Table 1). These mark the first detections of $\mathrm{NH}_{2} \mathrm{CDO}$, cisNHDCHO, trans-NHDCHO, and DNCO in the interstellar medium. The list of unblended lines can be found in the Appendix. Maps of the integrated line emission from representative lines from the different isotopologues towards source B are shown in Fig. 1. The emission of the different lines clearly arise from a similar compact region in the vicinity of IRAS16293B.

\footnotetext{
1 http://youngstars.nbi.dk/PILS/

2 http://cassis.irap.omp.eu/
}

Table 1. Number of lines used in the analysis of the isotopologues of $\mathrm{NH}_{2} \mathrm{CHO}$ and $\mathrm{HNCO}$ and column densities derived for $T_{\mathrm{ex}}=300 \mathrm{~K}$ and a source size of $0.5^{\prime \prime}$.

\begin{tabular}{lccc}
\hline \hline Species & \# of lines & $E_{\text {up }}(\mathrm{K})$ & $N\left(\mathrm{~cm}^{-2}\right)$ \\
\hline $\mathrm{NH}_{2} \mathrm{CDO}$ & 12 & $146-366$ & $2.1 \times 10^{14}$ \\
cis-NHDCHO & 11 & $146-307$ & $2.1 \times 10^{14}$ \\
trans-NHDCHO & 11 & $151-332$ & $1.8 \times 10^{14}$ \\
$\mathrm{NH}_{2}{ }^{13} \mathrm{CHO}$ & 10 & $152-428$ & $1.5 \times 10^{14}$ \\
${ }^{15} \mathrm{NH}_{2} \mathrm{CHO}$ & - & - & $\leq 1.0 \times 10^{14 a}$ \\
$\mathrm{NH}_{2} \mathrm{CH}{ }^{18} \mathrm{O}$ & - & - & $\leq 0.8 \times 10^{14 a}$ \\
$\mathrm{DNCO}$ & 4 & $150-751$ & $3.0 \times 10^{14}$ \\
$\mathrm{HN}{ }^{13} \mathrm{CO}$ & 8 & $127-532$ & $4.0 \times 10^{14}$ \\
$\mathrm{H}^{15} \mathrm{NCO}$ & - & - & $\leq 2.0 \times 10^{14 a}$ \\
$\mathrm{HNC}{ }^{18} \mathrm{O}$ & - & - & $\leq 1.5 \times 10^{14 a}$ \\
\hline
\end{tabular}

Notes. ${ }^{(a)} 3 \sigma$ upper limit.

A hole is observed in the maps owing to the absorptions that are produced against the strong continuum at the continuum peak position. For DNCO, the larger beam size for the observations of this transition masks the absorption. The spatial variations that are observed among the different species are probably due to different line excitation or line brightness. In particular, $\mathrm{HNCO}$ seems to be slightly more extended than $\mathrm{NH}_{2} \mathrm{CHO}$, but this is most likely due to the fact that the HNCO lines are particularly bright compared to the HNCO and formamide isotopologues.

To constrain the excitation temperatures and column densities of the different species, we produce a grid of synthetic spectra, assuming local thermodynamical equilibrium (LTE). We predict the spectra for different excitation temperatures between 100 and $300 \mathrm{~K}$ with a step of $25 \mathrm{~K}$ and for different column densities between $1 \times 10^{13}$ and $1 \times 10^{17} \mathrm{~cm}^{-2}$. First, the column density is roughly estimated using relatively large steps, then refined using smaller steps around the best-fit solution. We determine the best-fit model using a $\chi^{2}$ method, comparing the observed and synthetic spectra at $\pm 0.5 \mathrm{MHz}$ around the rest frequency of the predicted emission lines. We carefully check that the best-fit model does not predict any lines that are not observed in the spectra. For the deuterated forms, the models are in agreement with the observations for excitation temperatures between 100 and $300 \mathrm{~K}$. However, for $\mathrm{NH}_{2}{ }^{13} \mathrm{CHO}$ and $\mathrm{HN}^{13} \mathrm{CO}$, a model with a high excitation temperature better reproduces the observed emission than a model with a low excitation temperature (see Figs. B.4 and B.6). An excitation temperature of $300 \mathrm{~K}$ was consequently adopted for the analysis of the different isotopologues. This excitation temperature is similar to that derived for glycolaldehyde and ethylene glycol (Jørgensen et al. 2012, 2016), but higher than what is found for acetaldehyde, ethylene oxide, and propanal ( $\sim 125 \mathrm{~K}$, Lykke et al., in prep.). The derived column densities, assuming a linewidth of $1 \mathrm{~km} \mathrm{~s}^{-1}$ and a source size of $0.5^{\prime \prime}$ (Jørgensen et al. 2016; Lykke et al., in prep.), are summarized in Table 1. The uncertainties on the column densities are all estimated to be within a factor of 2 (including the uncertainty on both the excitation temperature and the baseline subtraction). The upper limits are estimated visually by a comparison of the synthetic spectra with the observations on the entire spectral range. Figure 2 shows three lines of each isotopologue with the best-fit model. The models for all the lines are shown in Appendix B.

The column densities of $\mathrm{NH}_{2}{ }^{13} \mathrm{CHO}$, and $\mathrm{HN}^{13} \mathrm{CO}$ are estimated to be $1.5 \times 10^{14} \mathrm{~cm}^{-2}$ and $4 \times 10^{14} \mathrm{~cm}^{-2}$, respectively. Assuming a ${ }^{12} \mathrm{C} /{ }^{13} \mathrm{C}$ ratio of 68 (Milam et al. 2005), the column densities for the main isotopologues of formamide and isocyanic acid are predicted to be $1 \times 10^{16} \mathrm{~cm}^{-2}$ and $3 \times 10^{16} \mathrm{~cm}^{-2}$. With 


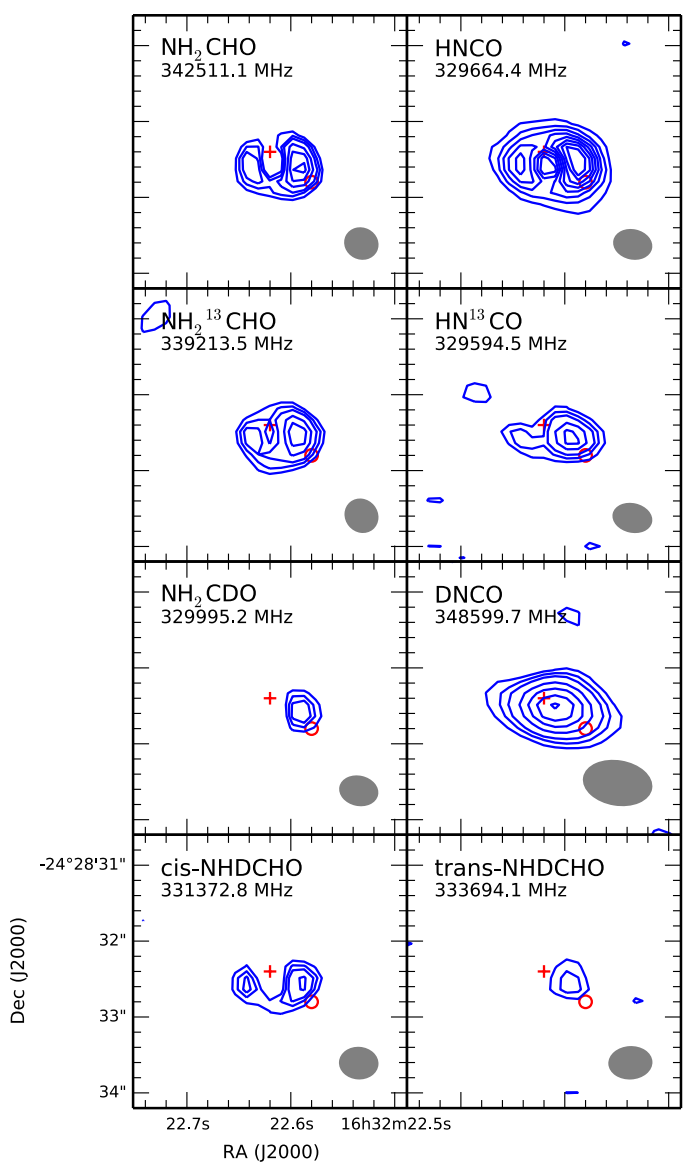

Fig. 1. Integrated intensity maps of $\mathrm{NH}_{2} \mathrm{CHO}, \mathrm{HNCO}$, and their isotopologues towards source B. The position of the continuum peak of source B is indicated with a red cross, while the position of where the spectrum was extracted is shown with a red circle. The beam sizes are shown in gray in the bottom right corner of each panel. The contour levels start for the main isotopologue of $\mathrm{HNCO}$ at $0.05 \mathrm{Jy} \mathrm{km} \mathrm{s}^{-1}$ with a step of $0.05 \mathrm{Jy} \mathrm{km} \mathrm{s}^{-1}$. For the other species, the levels are $0.02,0.03$, $0.04,0.06,0.08,0.1$, and $0.12 \mathrm{Jy} \mathrm{km} \mathrm{s}^{-1}$.

these column densities, several $\mathrm{NH}_{2} \mathrm{CHO}$ lines and all of the HNCO lines are overproduced, indicating that they are optically thick. The model of formamide is, however, in agreement with the few lines with the lowest opacities (see Figs. B.7 and B.8). $\mathrm{NH}_{2} \mathrm{CH}^{18} \mathrm{O}$ has also been searched for, but is not detected with a $3 \sigma$ upper limit of $8 \times 10^{13} \mathrm{~cm}^{-2}$. The non-detection of this isotopologue is consistent with the ${ }^{16} \mathrm{O} /{ }^{18} \mathrm{O}$ ratio of 560 in the interstellar medium (Wilson 1999), which gives $N\left(\mathrm{NH}_{2} \mathrm{CH}^{18} \mathrm{O}\right)=$ $2 \times 10^{13} \mathrm{~cm}^{-2}$. Similarly, $\mathrm{HNC}^{18} \mathrm{O}$ is not detected either with a $3 \sigma$ upper limit of $1.5 \times 10^{14} \mathrm{~cm}^{-2}$, which is consistent with its expected column density of $5 \times 10^{13} \mathrm{~cm}^{-2}$.

Using the column densities derived for the ${ }^{13} \mathrm{C}$ isotopologues and a standard ${ }^{12} \mathrm{C} /{ }^{13} \mathrm{C}$ ratio, the deuterium fractionation in $\mathrm{NH}_{2} \mathrm{CHO}$ is about $2 \%$ for the three deuterated forms and the DNCO/HNCO ratio is similar $(\sim 1 \%)$. If the ${ }^{12} \mathrm{C} /{ }^{13} \mathrm{C}$ ratio is lower $(\sim 30)$ as reported for glycolaldehyde by Jørgensen et al. (2016), the $\mathrm{D} / \mathrm{H}$ ratios of formamide and isocyanic acid would be about $4-5 \%$ and $2-3 \%$, respectively.

We also search for the ${ }^{15} \mathrm{~N}$ isotopologues of formamide and isocyanic acid. A couple of transitions could tentatively be assigned to ${ }^{15} \mathrm{NH}_{2} \mathrm{CHO}$, but these lines are close to the noise level and possibly blended with other species. For $\mathrm{H}^{15} \mathrm{NCO}$, the uncertainties on the frequencies of some of the transitions are rather large, preventing any firm detection. Based on a standard ${ }^{12} \mathrm{C} /{ }^{13} \mathrm{C}$
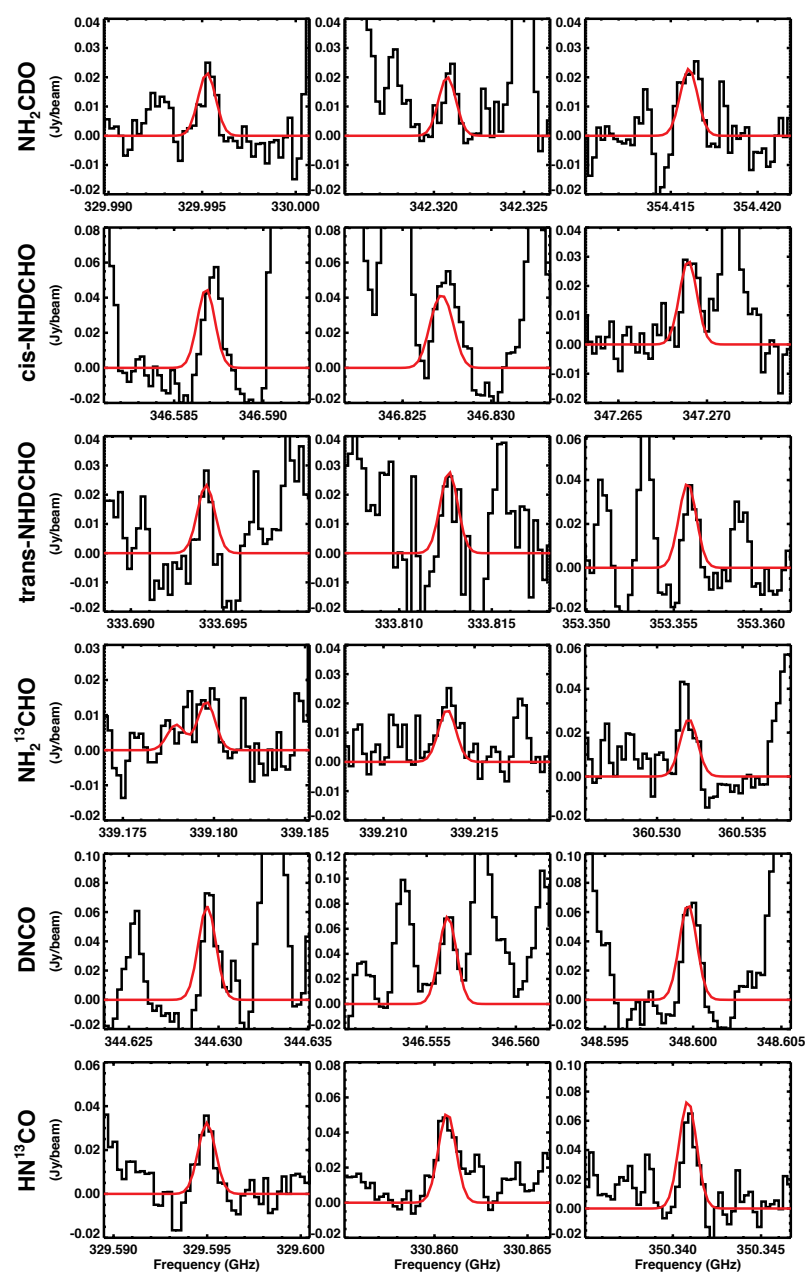

Fig. 2. Black: detected lines of $\mathrm{NH}_{2} \mathrm{CDO}$, cis-NHDCHO, trans$\mathrm{NHDCHO}, \mathrm{NH}_{2}^{13} \mathrm{CHO}, \mathrm{DNCO}$, and $\mathrm{HN}^{13} \mathrm{CO}$. Red: best-fit model.

ratio, lower limits of 100 and 138 are obtained for the ${ }^{14} \mathrm{~N} /{ }^{15} \mathrm{~N}$ ratios of formamide and $\mathrm{HNCO}$ respectively.

\section{Discussion and conclusion}

Our derived ratio in IRAS16293 for $\mathrm{HNCO} / \mathrm{NH}_{2} \mathrm{CHO}, \sim 3$, is consistent with the ratios found in warm sources in previous studies (Bisschop et al. 2007; Mendoza et al. 2014; López-Sepulcre et al. 2015). Thanks to our interferometric observations, we also confirm that these two species are spatially correlated. The deuterium fractionation ratios of these two molecules are also similar, reinforcing the hypothesis that they are chemically related. We discuss here possible scenarios for the formation of these species in the warm inner regions of protostars.

Assuming that the deuteration of formaldehyde in the region probed by the ALMA observations of formamide is similar to the value derived with single-dish observations $(\sim 15 \%$, Loinard et al. 2000), we can discuss the possibility for the gasphase formation mechanism proposed by Barone et al. (2015), $\mathrm{H}_{2} \mathrm{CO}+\mathrm{NH}_{2} \rightarrow \mathrm{NH}_{2} \mathrm{CHO}+\mathrm{H}$. According to this reaction, the deuterated form NHDCHO would result from the reaction between NHD and $\mathrm{H}_{2} \mathrm{CO}$, while $\mathrm{NH}_{2} \mathrm{CDO}$ would form from $\mathrm{NH}_{2}$ and HDCO. We would consequently expect a higher deuteration for $\mathrm{NH}_{2} \mathrm{CDO}$, compared to the observations, unless the reaction between $\mathrm{NH}_{2}$ and HDCO leads more efficiently to $\mathrm{NH}_{2} \mathrm{CHO}$ 
and $\mathrm{D}$, compared to $\mathrm{NH}_{2} \mathrm{CDO}$ and $\mathrm{H}$. Theoretical or experimental studies of the branching ratios of these reactions would be needed to rule out this scenario. The determination of the $\mathrm{HDCO} / \mathrm{H}_{2} \mathrm{CO}$ ratio from the PILS survey is also necessary. Nevertheless, it should be noted that, so far, there is no proposed scenario in the gas phase that could explain the correlation with HNCO.

Although it was recently shown that $\mathrm{NH}_{2} \mathrm{CHO}$ does not form by hydrogenation of HNCO on grain surfaces (Noble et al. 2015), several other proposed mechanisms exist in the literature. Both species can be formed through barrierless reactions in ices through $\mathrm{NH}+\mathrm{CO} \rightarrow \mathrm{HNCO}$ and $\mathrm{NH}_{2}+\mathrm{H}_{2} \mathrm{CO} \rightarrow$ $\mathrm{NH}_{2} \mathrm{CHO}+\mathrm{H}$, as demonstrated experimentally (Fedoseev et al. 2015, 2016). Alternatively, both species are formed through ion bombardment of $\mathrm{H}_{2} \mathrm{O}: \mathrm{CH}_{4}: \mathrm{N}_{2}$ mixtures (Kaňuchová et al. 2016) or UV irradiation of $\mathrm{CO}: \mathrm{NH}_{3}: \mathrm{CH}_{3} \mathrm{OH}$ and/or HNCO mixtures (e.g. Demyk et al. 1998; Raunier et al. 2004; Jones et al. 2011; Henderson \& Gudipati 2015). Quantitative gas-grain modeling under conditions representative of IRAS16293 are needed to assess which of these grain surface routes dominates.

Ultimately, the $\mathrm{HNCO}$ and $\mathrm{NH}_{2} \mathrm{CHO}$ deuterium fractionation level and pattern may also hold a clue to their formation routes. A particularly interesting result is that the three singly deuterated forms of formamide are found with similar abundances in IRAS16293. Contrary to the - $\mathrm{CH}$ functional group that is not affected by hydrogen isotope exchanges, the hydroxyl $(-\mathrm{OH})$ and amine $(-\mathrm{NH})$ groups are expected to establish hydrogen bonds and equilibrate with water (Faure et al. 2015). This mechanism was proposed to explain the different $\mathrm{CH}_{3} \mathrm{OD} / \mathrm{CH}_{3} \mathrm{OH}(\sim 1.8 \%)$ and $\mathrm{CH}_{2} \mathrm{DOH} / \mathrm{CH}_{3} \mathrm{OH}(\sim 37 \%)$ ratios derived in IRAS16293 (Parise et al. 2006), as the water deuterium fractionation of water in the upper layers of the grain mantles, where complex organic molecules form, is about a few percent (Coutens et al. 2012, 2013; Furuya et al. 2016). We do not see these types of differences for formamide, for which all forms show a deuterium fractionation similar to the $\mathrm{CH}_{3} \mathrm{OD} / \mathrm{CH}_{3} \mathrm{OH}$ ratio and water. The deuterium fractionation of methanol from the PILS data needs to be investigated to know if the different deuterium fractionation ratios of the $-\mathrm{CH}$ and $-\mathrm{OH}$ groups are also observed at small scales.

In conclusion, in this Letter we present the first detection of the three singly deuterated forms of formamide and DNCO. The similar deuteration of these species and their similar spatial distributions favors the formation of these two species on grain surfaces. Further studies are, however, needed to rule out gas phase routes. These detections illustrate the strength of ALMA, and large spectral surveys such as PILS in particular, for the detections of deuterated complex molecules. Determinations of the deuterium fractionation for more complex molecules will help to constrain their formation pathways. The search for deuterated formamide in more sources is needed to reveal how variable the deuteration of formamide is, and if the similarity of the abundances of the three deuterated forms is common.

Acknowledgements. The authors thank Gleb Fedoseev and Harold Linnartz for fruitful discussions. This paper makes use of the following ALMA data: ADS/JAO.ALMA\#2013.1.00278.S. ALMA is a partnership of ESO (representing its member states), NSF (USA) and NINS (Japan), together with NRC (Canada) and NSC and ASIAA (Taiwan), in cooperation with the Republic of Chile. The Joint ALMA Observatory is operated by ESO, AUI/NRAO and NAOJ. The work of A.C. was funded by an STFC grant. A.C. thanks the COST action CM1401 Our Astrochemical History for additional financial support. The group of J.K.J. acknowledges support from a Lundbeck Foundation Group Leader Fellowship, as well as the European Research Council (ERC) under the European Union's Horizon 2020 research and innovation programme (grant agreement No 646908) through ERC Consolidator Grant S4F. Research at the Centre for Star and Planet Formation is funded by the Danish National Research Foundation. The group of E.v.D. acknowledges A-ERC grant 291141 CHEMPLAN.

\section{References}

Adande, G. R., Woolf, N. J., \& Ziurys, L. M. 2013, Astrobiol., 13, 439 Barone, V., Latouche, C., Skouteris, D., et al. 2015, MNRAS, 453, L31 Bisschop, S. E., Jørgensen, J. K., van Dishoeck, E. F., \& de Wachter, E. B. M. 2007, A\&A, 465, 913

Biver, N., Bockelée-Morvan, D., Debout, V., et al. 2014, A\&A, 566, L5 Blanco, S., Lopez, J. C., Lessari, A., \& Alonso, J. L. 2006, J. Am. Chem. Soc., 128, 12111

Bockelée-Morvan, D., Lis, D. C., Wink, J. E., et al. 2000, A\&A, 353, 1101

Ceccarelli, C., Caselli, P., Herbst, E., Tielens, A. G. G. M., \& Caux, E. 2007, Protostars and Planets V, 47

Corby, J. F., Jones, P. A., Cunningham, M. R., et al. 2015, MNRAS, 452, 3969

Coutens, A., Vastel, C., Caux, E., et al. 2012, A\&A, 539, A132

Coutens, A., Vastel, C., Cazaux, S., et al. 2013, A\&A, 553, A75

Demyk, K., Dartois, E., D'Hendecourt, L., et al. 1998, A\&A, 339, 553

Demyk, K., Bottinelli, S., Caux, E., et al. 2010, A\&A, 517, A17

Faure, A., Faure, M., Theulé, P., Quirico, E., \& Schmitt, B. 2015, A\&A, 584, A98

Fedoseev, G., Ioppolo, S., Zhao, D., Lamberts, T., \& Linnartz, H. 2015, MNRAS, 446, 439

Fedoseev, G., Chuang, K.-J., van Dishoeck, E. F., Ioppolo, S., \& Linnartz, H. 2016, MNRAS, in press

Furuya, K., van Dishoeck, E. F., \& Aikawa, Y. 2016, A\&A, 586, A127

Gardner, F. F., Godfrey, P. D., \& Williams, D. R. 1980, MNRAS, 193, 713

Garrod, R. T. 2013, ApJ, 765, 60

Goesmann, F., Rosenbauer, H., Bredehöft, J. H., et al. 2015, Science, 349, 020689

Henderson, B. L., \& Gudipati, M. S. 2015, ApJ, 800, 66

Hirota, E., Sugisaki, R., Nielsen, C. J., \& Sørensen, G. O. 1974, J. Mol. Spectr., 49, 251

Hocking, W. H., Gerry, M. C. L., \& Winnewisser, G. 1975, Can. J. Phys., 53, 1869

Jones, B. M., Bennett, C. J., \& Kaiser, R. I. 2011, ApJ, 734, 78

Jørgensen, J. K., Favre, C., Bisschop, S. E., et al. 2012, ApJ, 757, L4

Jørgensen, J. K., van der Wiel, M. H. D., Coutens, A., et al. 2016, A\&A, submitted

Kahane, C., Ceccarelli, C., Faure, A., \& Caux, E. 2013, ApJ, 763, L38

Kaňuchová, Z., Urso, R. G., Baratta, G. A., et al. 2016, A\&A, 585, A155

Kryvda, A. V., Gerasimov, V. G., Dyubko, S. F., Alekseev, E. A., \& Motiyenko, R. A. 2009, J. Mol. Spectr., 254, 28

Kukolich, S. G., \& Nelson, A. C. 1971, Chem. Phys. Lett., 11, 383

Kurland, R. J., \& Bright Wilson, Jr., E. 1957, J. Chem. Phys., 27, 585

Kutsenko, A. S., Motiyenko, R. A., Margulès, L., \& Guillemin, J.-C. 2013, A\&A 549, A128

Lapinov, A. V., Golubiatnikov, G. Y., Markov, V. N., \& Guarnieri, A. 2007, Astron. Lett., 33, 121

Loinard, L., Castets, A., Ceccarelli, C., et al. 2000, A\&A, 359, 1169

López-Sepulcre, A., Jaber, A. A., Mendoza, E., et al. 2015, MNRAS, 449, 2438

Mendoza, E., Lefloch, B., López-Sepulcre, A., et al. 2014, MNRAS, 445, 151

Milam, S. N., Savage, C., Brewster, M. A., Ziurys, L. M., \& Wyckoff, S. 2005 , ApJ, 634, 1126

Moskienko, E. M., \& Dyubko, S. F. 1991, Radiophys. Quant. Electron., 34, 181

Motiyenko, R. A., Tercero, B., Cernicharo, J., \& Margulès, L. 2012, A\&A, 548, A71

Müller, H. S. P., Thorwirth, S., Roth, D. A., \& Winnewisser, G. 2001, A\&A, 370, L49

Müller, H. S. P., Schlöder, F., Stutzki, J., \& Winnewisser, G. 2005, J. Mol. Struct., 742,215

Muller, S., Beelen, A., Black, J. H., et al. 2013, A\&A, 551, A109

Niedenhoff, M., Yamada, K. M. T., Belov, S. P., \& Winnewisser, G. 1995, J. Mol. Spectr., 174, 151

Noble, J. A., Theule, P., Congiu, E., et al. 2015, A\&A, 576, A91

Parise, B., Ceccarelli, C., Tielens, A. G. G. M., et al. 2006, A\&A, 453, 949

Pickett, H. M., Poynter, R. L., Cohen, E. A., et al. 1998, J. Quant. Spectr. Rad. Transf., 60, 883

Raunier, S., Chiavassa, T., Duvernay, F., et al. 2004, A\&A, 416, 165

Redondo, P., Barrientos, C., \& Largo, A. 2014a, ApJ, 793, 32

Redondo, P., Barrientos, C., \& Largo, A. 2014b, ApJ, 780, 18

Richard, C., Margulès, L., Caux, E., et al. 2013, A\&A, 552, A117

Saladino, R., Crestini, C., Pino, S., Costanzo, G., \& Di Mauro, E. 2012, Physics of Life Reviews, 9, 84

Vorob'eva, E. M., \& Dyubko, S. F. 1994, Radiophys. Quant. Electron., 37, 155

Wilson, T. L. 1999, Rep. Progr. Phys., 62, 143

Yamaguchi, T., Takano, S., Watanabe, Y., et al. 2012, PASJ, 64, 105 


\section{Appendix A: Spectroscopic data}

A list of unblended and optically thin lines used in the analysis is presented in Table A.1. The spectroscopic data for $\mathrm{NH}_{2} \mathrm{CHO} v=0, \mathrm{NH}_{2} \mathrm{CHO} v_{12}=1, \mathrm{NH}_{2}{ }^{13} \mathrm{CHO},{ }^{15} \mathrm{NH}_{2} \mathrm{CHO}$, $\mathrm{NH}_{2} \mathrm{CH}^{18} \mathrm{O}$, $\mathrm{NH}_{2} \mathrm{CDO}$, cis-NHDCHO, trans-NHDCHO (Kurland \& Bright Wilson 1957; Kukolich \& Nelson 1971; Hirota et al. 1974; Gardner et al. 1980; Moskienko \& Dyubko 1991; Vorob'eva \& Dyubko 1994; Blanco et al. 2006; Kryvda et al. 2009; Motiyenko et al. 2012; Kutsenko et al. 2013) and HNCO (Kukolich \& Nelson 1971; Hocking et al. 1975; Niedenhoff et al. 1995; Lapinov et al. 2007) come from the Cologne Database for Molecular Spectroscopy (CMDS, Müller et al. 2001, 2005), while the data for DNCO, $\mathrm{HN}^{13} \mathrm{CO}, \mathrm{H}^{15} \mathrm{NCO}$ and $\mathrm{HNC}^{18} \mathrm{O}$ (Hocking et al. 1975) are taken from the Jet Propulsion Laboratory (JPL) database
(Pickett et al. 1998). It should be noted that there are significant differences for the predicted frequencies of the main isotopologue of $\mathrm{NH}_{2} \mathrm{CHO}$ between CDMS and JPL ( $\left.>1 \mathrm{MHz}\right)$. A better agreement is found with the observations for the most recent entry in CDMS. For some of the HNCO isotopologues, there is a lack of published spectroscopic data at high frequencies. In particular for $\mathrm{H}^{15} \mathrm{NCO}$, the range of uncertainty for some of the frequencies is quite high. As the $\mathrm{HN}^{13} \mathrm{CO}$ transitions all appeared slightly shifted, compared to the observations, we applied a correction of $+0.5 \mathrm{MHz}$ to model the lines.

The column densities of the formamide isotopologues given in Table 1 were corrected by a factor of 1.5 to take into account the contribution of the vibrational states for an excitation temperature of $300 \mathrm{~K}$. 
Table A.1. Detected lines of $\mathrm{NH}_{2} \mathrm{CHO}, \mathrm{HNCO}$ and their isotopologues used in the analysis.

\begin{tabular}{|c|c|c|c|c|c|}
\hline Species & Transition & $\begin{array}{c}\text { Frequency } \\
(\mathrm{MHz})\end{array}$ & $\begin{array}{l}E_{\mathrm{up}} \\
(\mathrm{K})\end{array}$ & $\begin{array}{c}A_{\mathrm{ij}} \\
\left(\mathrm{s}^{-1}\right)\end{array}$ & $g_{\text {up }}$ \\
\hline $\mathrm{NH}_{2} \mathrm{CDO}$ & $\left(\begin{array}{lllll}17 & 0 & 17-16 & 0 & 16\end{array}\right)$ & 329995.2 & 145.6 & $2.64 \times 10^{-3}$ & 105 \\
\hline $\mathrm{NH}_{2} \mathrm{CDO}$ & $(1697-1596)$ & 333363.6 & 308.9 & $1.87 \times 10^{-3}$ & 99 \\
\hline $\mathrm{NH}_{2} \mathrm{CDO}$ & $(1698-1597)$ & 333363.6 & 308.9 & $1.87 \times 10^{-3}$ & 99 \\
\hline $\mathrm{NH}_{2} \mathrm{CDO}$ & (16 7 10-15 79 ) & 333696.6 & 240.7 & $2.22 \times 10^{-3}$ & 99 \\
\hline $\mathrm{NH}_{2} \mathrm{CDO}$ & (16 $79-1578)$ & 333696.6 & 240.7 & $2.22 \times 10^{-3}$ & 99 \\
\hline $\mathrm{NH}_{2} \mathrm{CDO}$ & $(164$ 13-15 4 12) & 335234.9 & 170.5 & $2.61 \times 10^{-3}$ & 99 \\
\hline $\mathrm{NH}_{2} \mathrm{CDO}$ & $(16313-15312)$ & 342320.7 & 156.9 & $2.86 \times 10^{-3}$ & 99 \\
\hline $\mathrm{NH}_{2} \mathrm{CDO}$ & 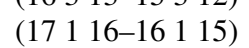 & 351988.3 & 158.1 & $3.18 \times 10^{-3}$ & 105 \\
\hline $\mathrm{NH}_{2} \mathrm{CDO}$ & $(17107-16106)$ & 354151.5 & 366.4 & $2.15 \times 10^{-3}$ & 105 \\
\hline $\mathrm{NH}_{2} \mathrm{CDO}$ & 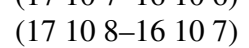 & 354151.5 & 366.4 & $2.15 \times 10^{-3}$ & 105 \\
\hline $\mathrm{NH}_{2} \mathrm{CDO}$ & $(1798-1697)$ & 354257.0 & 325.9 & $2.37 \times 10^{-3}$ & 105 \\
\hline $\mathrm{NH}_{2} \mathrm{CDO}$ & (1799-1698) & 354257.0 & 325.9 & $2.37 \times 10^{-3}$ & 105 \\
\hline $\mathrm{NH}_{2} \mathrm{CDO}$ & (17 $810-1689)$ & 354416.0 & 289.6 & $2.56 \times 10^{-3}$ & 105 \\
\hline $\mathrm{NH}_{2} \mathrm{CDO}$ & $(1789-1688)$ & 354416.0 & 289.6 & $2.56 \times 10^{-3}$ & 105 \\
\hline $\mathrm{NH}_{2} \mathrm{CDO}$ & $(17711-16710)$ & 354661.3 & 257.7 & $2.74 \times 10^{-3}$ & 105 \\
\hline $\mathrm{NH}_{2} \mathrm{CDO}$ & $(17710-1679)$ & 354661.3 & 257.7 & $2.74 \times 10^{-3}$ & 105 \\
\hline $\mathrm{NH}_{2} \mathrm{CDO}$ & $(175$ 12-16 5 11) & 355800.2 & 206.7 & $3.04 \times 10^{-3}$ & 105 \\
\hline $\mathrm{NH}_{2} \mathrm{CDO}$ & $(174$ 13-16 4 12) & 357938.5 & 187.8 & $3.20 \times 10^{-3}$ & 105 \\
\hline cis-NHDCHO & $(163$ 13-15 3 12) & 331372.8 & 156.0 & $2.59 \times 10^{-3}$ & 99 \\
\hline cis-NHDCHO & $(16214-15213)$ & 337248.5 & 146.0 & $2.79 \times 10^{-3}$ & 99 \\
\hline cis-NHDCHO & $(17216-16215)$ & 340520.3 & 158.0 & $2.87 \times 10^{-3}$ & 105 \\
\hline cis-NHDCHO & 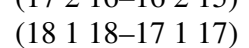 & 344878.9 & 160.8 & $3.02 \times 10^{-3}$ & 111 \\
\hline cis-NHDCHO & $(17810-1689)$ & 346444.0 & 306.6 & $2.39 \times 10^{-3}$ & 105 \\
\hline cis-NHDCHO & $(1789-1688)$ & 346444.0 & 306.6 & $2.39 \times 10^{-3}$ & 105 \\
\hline cis-NHDCHO & $(17711-16710)$ & 346586.8 & 269.8 & $2.56 \times 10^{-3}$ & 105 \\
\hline cis-NHDCHO & $(17710-1679)$ & 346586.8 & 269.8 & $2.56 \times 10^{-3}$ & 105 \\
\hline cis-NHDCHO & $(17612-16611)$ & 346826.8 & 238.0 & $2.70 \times 10^{-3}$ & 105 \\
\hline cis-NHDCHO & $(17611-16610)$ & 346827.5 & 238.0 & $2.70 \times 10^{-3}$ & 105 \\
\hline cis-NHDCHO & $(173$ 15-16 314$)$ & 347115.8 & 172.0 & $2.99 \times 10^{-3}$ & 105 \\
\hline cis-NHDCHO & $(17512-16511)$ & 347268.9 & 211.1 & $2.83 \times 10^{-3}$ & 105 \\
\hline cis-NHDCHO & $(174$ 14-16 4 13) & 347827.8 & 189.2 & $2.94 \times 10^{-3}$ & 105 \\
\hline cis-NHDCHO & (17 3 14-16 3 13) & 353047.5 & 173.0 & $3.15 \times 10^{-3}$ & 105 \\
\hline trans-NHDCHO & $(1789-1688)$ & 333628.6 & 332.4 & $2.14 \times 10^{-3}$ & 105 \\
\hline trans-NHDCHO & (17 $810-1689)$ & 333628.6 & 332.4 & $2.14 \times 10^{-3}$ & 105 \\
\hline trans-NHDCHO & $(177$ 11-16 7 10) & 333694.1 & 288.3 & $2.28 \times 10^{-3}$ & 105 \\
\hline trans-NHDCHO & $(177$ 10-16 79$)$ & 333694.1 & 288.3 & $2.28 \times 10^{-3}$ & 105 \\
\hline trans-NHDCHO & $(17612-16611)$ & 333812.6 & 250.1 & $2.41 \times 10^{-3}$ & 105 \\
\hline trans-NHDCHO & $(17611-16610)$ & 333812.7 & 250.1 & $2.41 \times 10^{-3}$ & 105 \\
\hline trans-NHDCHO & $(174$ 14-16 4 13) & 334403.2 & 191.4 & $2.61 \times 10^{-3}$ & 105 \\
\hline trans-NHDCHO & 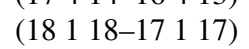 & 336945.3 & 157.3 & $2.82 \times 10^{-3}$ & 111 \\
\hline trans-NHDCHO & 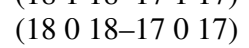 & 338818.4 & 156.9 & $2.87 \times 10^{-3}$ & 111 \\
\hline trans-NHDCHO & 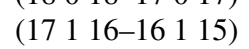 & 338878.8 & 150.6 & $2.86 \times 10^{-3}$ & 105 \\
\hline trans-NHDCHO & $(187$ 12-17 711$)$ & 353355.8 & 305.2 & $2.77 \times 10^{-3}$ & 111 \\
\hline trans-NHDCHO & $(18711-17710)$ & 353355.8 & 305.2 & $2.77 \times 10^{-3}$ & 111 \\
\hline trans-NHDCHO & $\left(\begin{array}{l}185 \\
5\end{array}\right.$ & 353758.4 & 234.7 & $3.02 \times 10^{-3}$ & 111 \\
\hline trans-NHDCHO & $\left(\begin{array}{llll}18 & 3 & 16 & -17 \\
3 & 15\end{array}\right)$ & 354028.8 & 187.8 & $3.19 \times 10^{-3}$ & 111 \\
\hline trans-NHDCHO & $(184$ 15-17 414$)$ & 354185.9 & 208.4 & $3.13 \times 10^{-3}$ & 111 \\
\hline $\mathrm{NH}_{2}{ }^{13} \mathrm{CHO}$ & $(16106-15105)$ & 339170.1 & 427.9 & $1.75 \times 10^{-3}$ & 33 \\
\hline $\mathrm{NH}_{2}{ }^{13} \mathrm{CHO}$ & $(16107-15106)$ & 339170.1 & 427.9 & $1.75 \times 10^{-3}$ & 33 \\
\hline $\mathrm{NH}_{2}{ }^{13} \mathrm{CHO}$ & $(1697-1596)$ & 339179.6 & 373.0 & $1.97 \times 10^{-3}$ & 33 \\
\hline $\mathrm{NH}_{2}{ }^{13} \mathrm{CHO}$ & $(1698-1597)$ & 339179.6 & 373.0 & $1.97 \times 10^{-3}$ & 33 \\
\hline $\mathrm{NH}_{2}{ }^{13} \mathrm{CHO}$ & $(1688-1587)$ & 339213.5 & 323.8 & $2.16 \times 10^{-3}$ & 33 \\
\hline $\mathrm{NH}_{2}{ }^{13} \mathrm{CHO}$ & $(1689-1588)$ & 339213.5 & 323.8 & $2.16 \times 10^{-3}$ & 33 \\
\hline $\mathrm{NH}_{2}{ }^{13} \mathrm{CHO}$ & $(16511-15510)$ & 339672.1 & 210.9 & $2.61 \times 10^{-3}$ & 33 \\
\hline $\mathrm{NH}_{2}{ }^{13} \mathrm{CHO}$ & $(164$ 13-15 4 12) & 340090.4 & 184.9 & $2.72 \times 10^{-3}$ & 33 \\
\hline $\mathrm{NH}_{2}{ }^{13} \mathrm{CHO}$ & $(16412-15411)$ & 340273.4 & 184.9 & $2.73 \times 10^{-3}$ & 33 \\
\hline $\mathrm{NH}_{2}{ }^{13} \mathrm{CHO}$ & 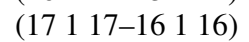 & 342156.0 & 151.5 & $2.95 \times 10^{-3}$ & 35 \\
\hline $\mathrm{NH}_{2}{ }^{13} \mathrm{CHO}$ & $(1798-1697)$ & 360396.3 & 390.3 & $2.49 \times 10^{-3}$ & 35 \\
\hline $\mathrm{NH}_{2}{ }^{13} \mathrm{CHO}$ & (1799-1698) & 360396.3 & 390.3 & $2.49 \times 10^{-3}$ & 35 \\
\hline $\mathrm{NH}_{2}{ }^{13} \mathrm{CHO}$ & $(17711-16710)$ & 360531.8 & 297.7 & $2.88 \times 10^{-3}$ & 35 \\
\hline
\end{tabular}

Notes. This list only includes optically thin and unblended lines. 
A. Coutens et al.: The ALMA-PILS survey: First detections of deuterated formamide and deuterated isocyanic acid

Table A.1. continued.

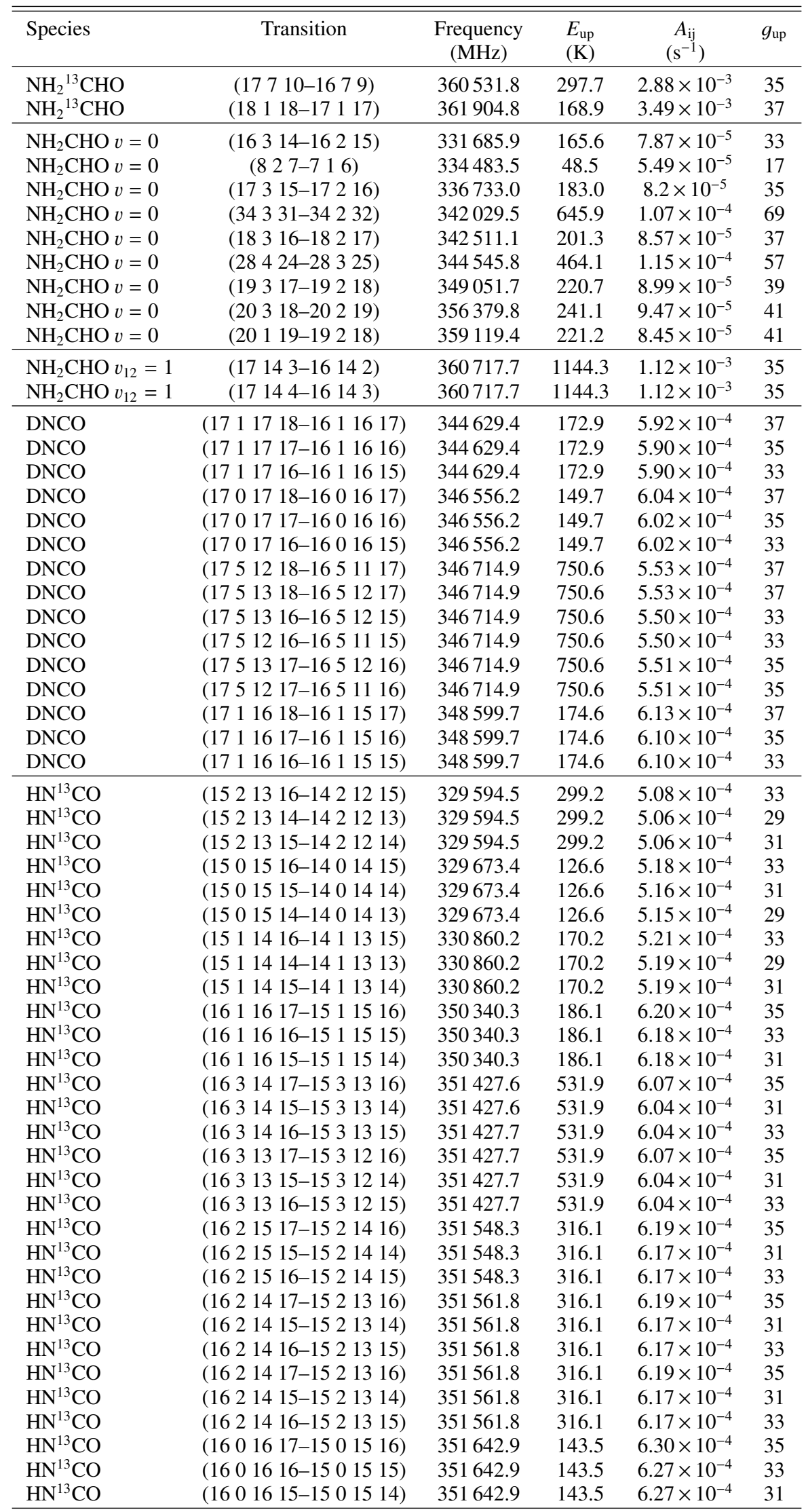




\section{Appendix B: Additional figures}
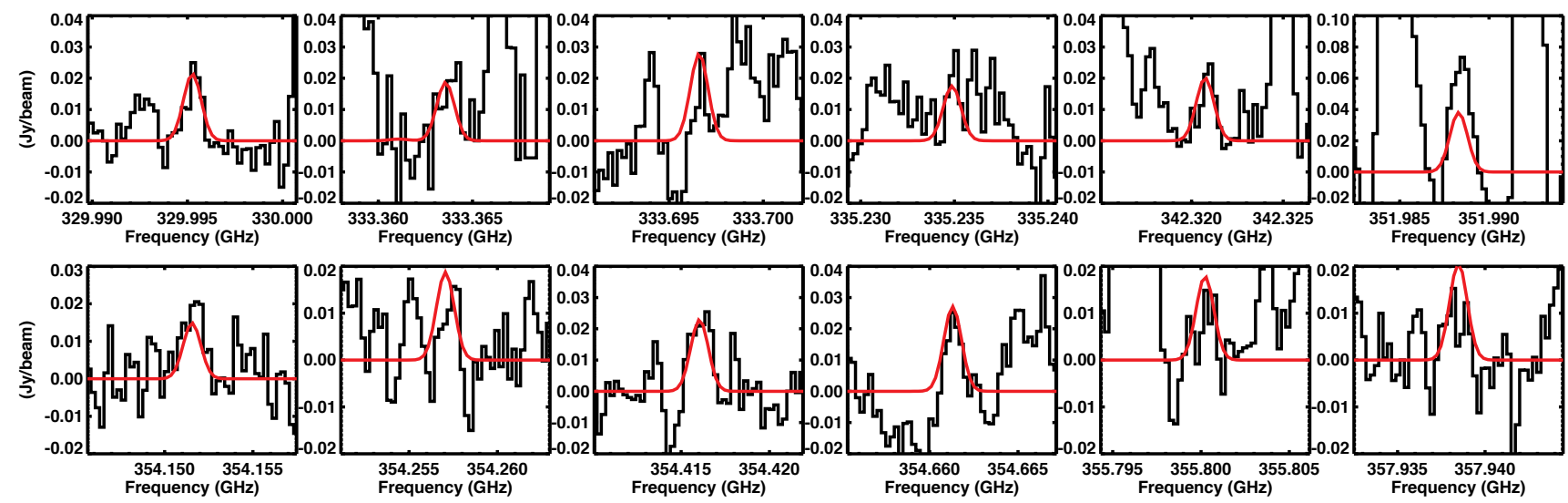

Fig. B.1. Black: detected lines of $\mathrm{NH}_{2} \mathrm{CDO}$. Red: best-fit model for $T_{\mathrm{ex}}=300 \mathrm{~K}$.
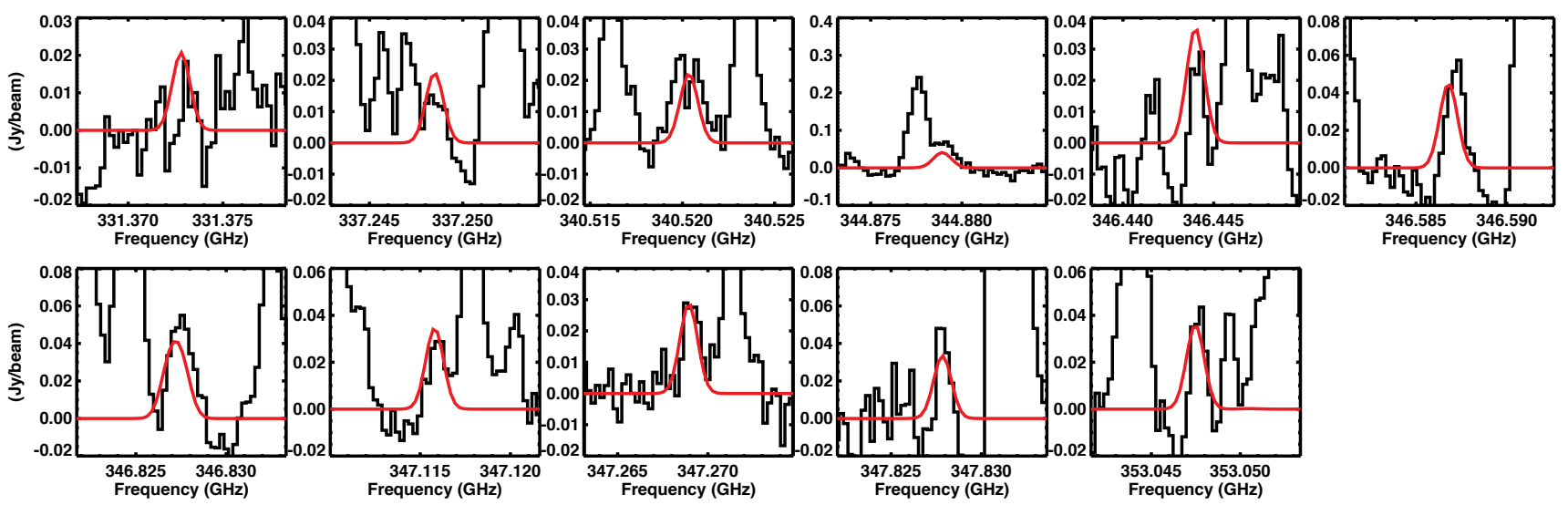

Fig. B.2. Black: detected lines of cis-NHDCHO. Red: best-fit model for $T_{\mathrm{ex}}=300 \mathrm{~K}$.
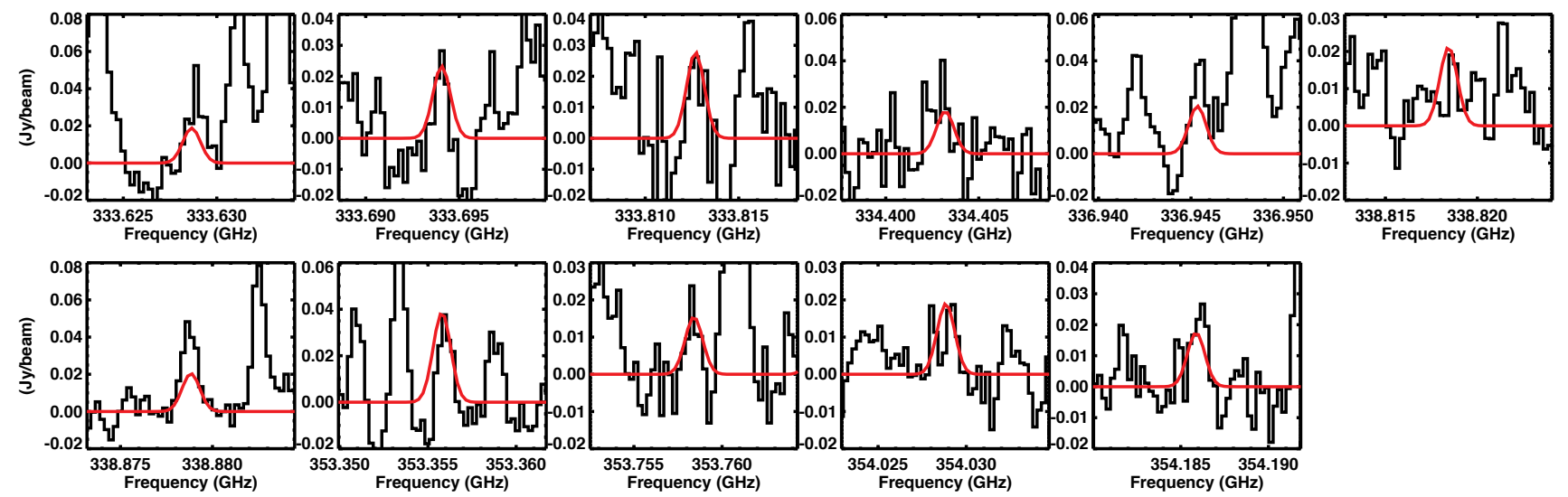

Fig. B.3. Black: detected lines of trans-NHDCHO. Red: best-fit model for $T_{\mathrm{ex}}=300 \mathrm{~K}$. 

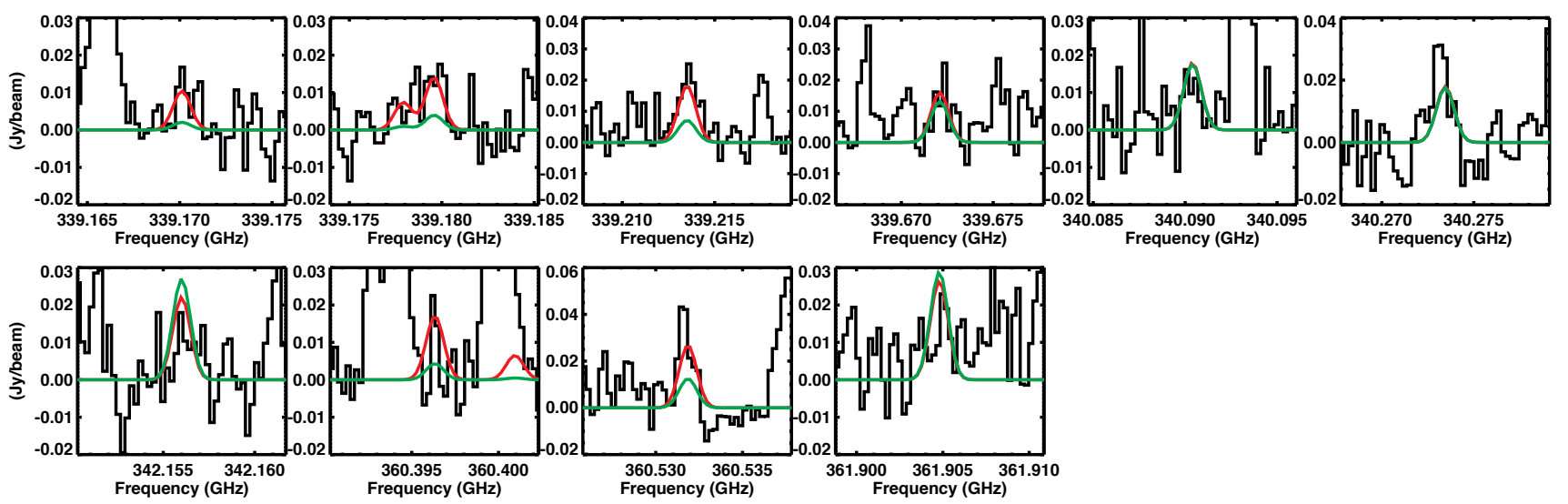

Fig. B.4. Black: detected lines of $\mathrm{NH}_{2}^{13} \mathrm{CHO}$. Red: best-fit model for $T_{\mathrm{ex}}=300 \mathrm{~K}$. Green: best-fit model for $T_{\mathrm{ex}}=100 \mathrm{~K}$.

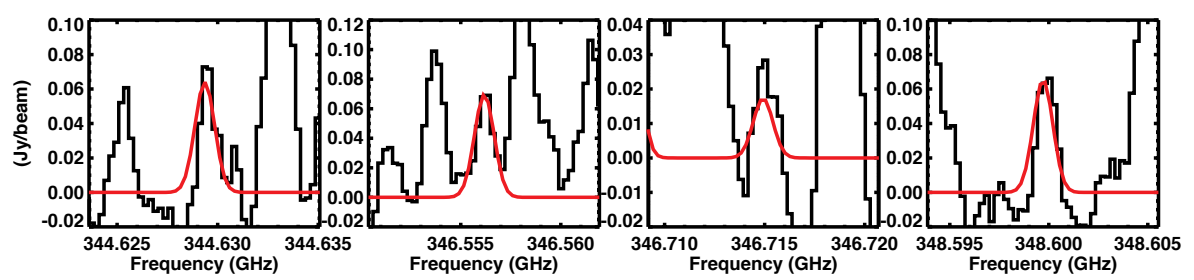

Fig. B.5. Black: detected lines of DNCO. Red: best-fit model for $T_{\mathrm{ex}}=300 \mathrm{~K}$.
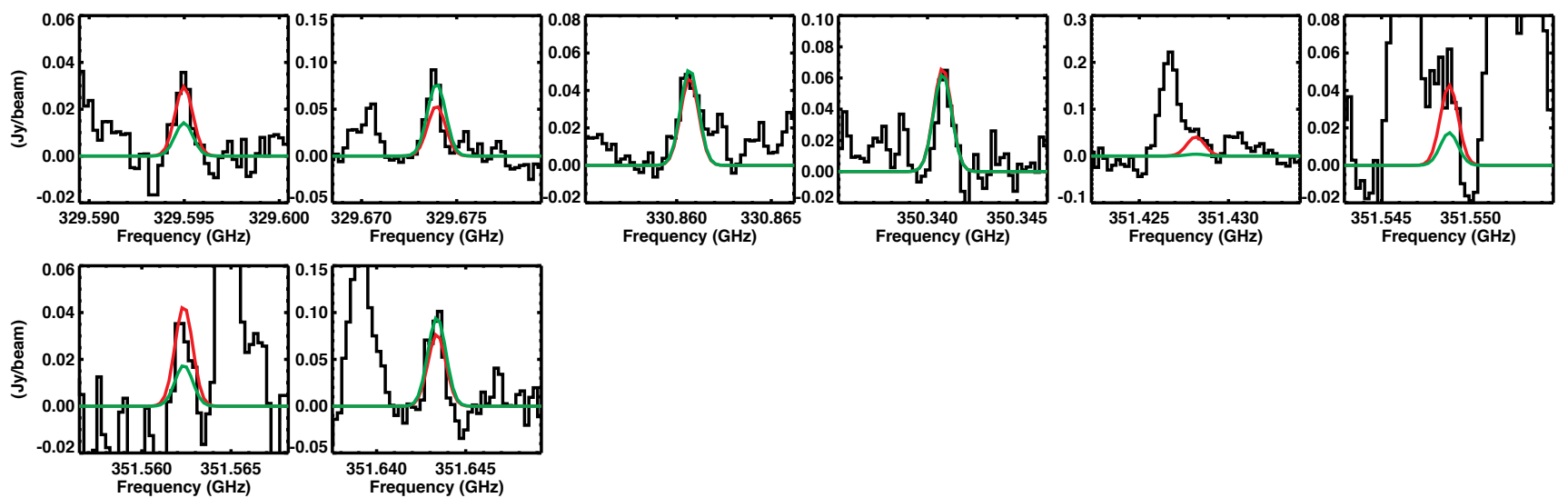

Fig. B.6. Black: detected lines of $\mathrm{HN}^{13} \mathrm{CO}$. Red: best-fit model for $T_{\mathrm{ex}}=300 \mathrm{~K}$. Green: best-fit model for $T_{\mathrm{ex}}=100 \mathrm{~K}$.
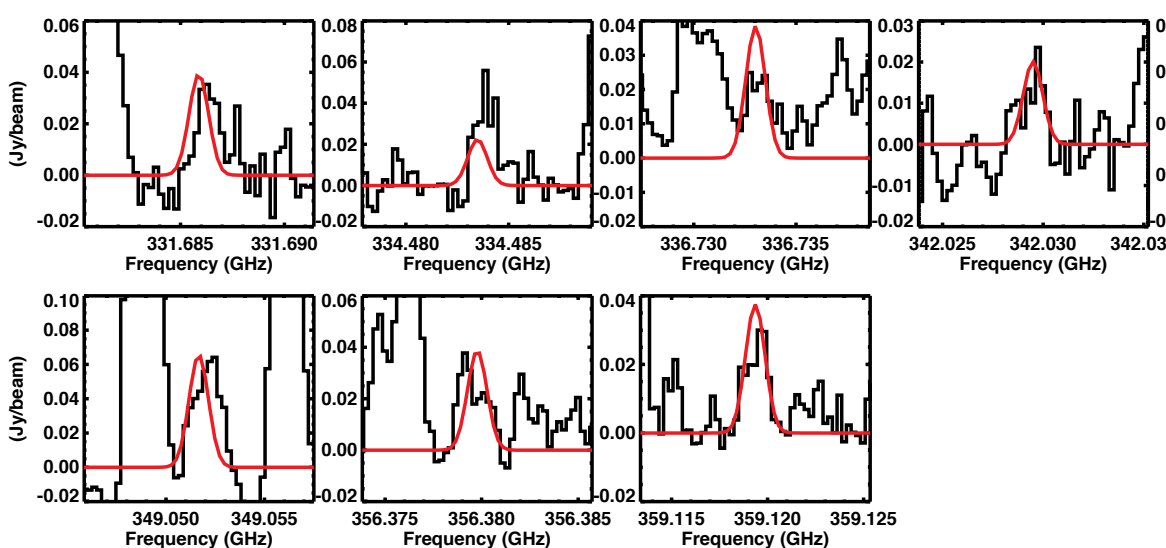

Fig. B.7. Black: lines of $\mathrm{NH}_{2} \mathrm{CHO} v=0$ with the lowest opacities. Red: model based on the analysis of the $\mathrm{NH}_{2}^{13} \mathrm{CHO}^{\text {lines and a }}{ }^{12} \mathrm{C} /{ }^{13} \mathrm{C}$ ratio equal to 68 . 
A\&A 590, L6 (2016)

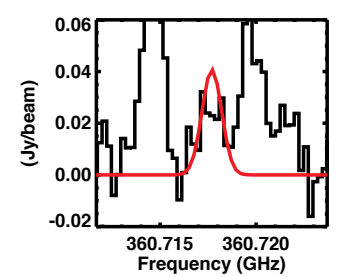

Fig. B.8. Black: line of $\mathrm{NH}_{2} \mathrm{CHO} v_{12}=1$ with the lowest opacity. Red: model based on the analysis of the $\mathrm{NH}_{2}^{13} \mathrm{CHO}$ lines and a ${ }^{12} \mathrm{C} /{ }^{13} \mathrm{C}$ ratio equal to 68 . 